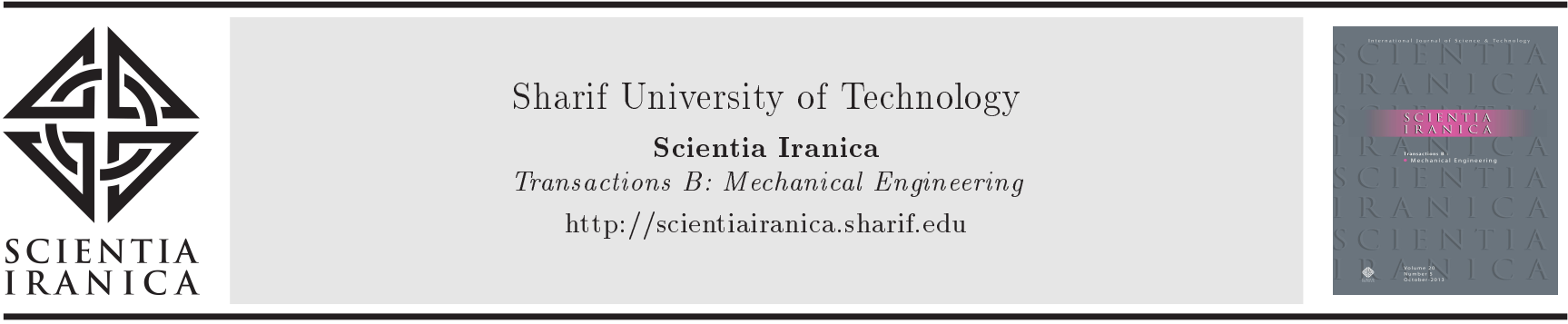

\title{
Nonlinear oscillations of viscoelastic microcantilever beam based on modified strain gradient theory
}

\author{
F. Taheran, M.T. Ahmadian*, and K. Firoozbakhsh \\ School of Mechanical Engineering, Sharif University of Technology, Tehran, P.O. Box 11155-9567, Iran.
}

Received 29 July 2019; received in revised form 18 December 2019; accepted 20 April 2020

\section{KEYWORDS}

Microcantilever beam;

Multiple time scale

method;

Nonlinear free

vibration;

Strain gradient

theory;

Viscoelastic material.

\begin{abstract}
A viscoelastic microcantilever beam is analytically analyzed based on the modified strain gradient theory. Kelvin-Voigt scheme is used to model beam viscoelasticity. By applying Euler-Bernoulli inextensibility of the centerline condition based on Hamilton's principle, the nonlinear equation of motion and the related boundary conditions are derived from shortening effect theory and discretized by Galerkin method. Inner damping, nonlinear curvature effect, and nonlinear inertia terms are also taken into account. In the present study, the generalized derived formulation allows modeling any nonlinear combination such as nonlinear terms that arise due to inertia, damping, and stiffness, as well as modeling the size effect using modified coupled stress or modified strain gradient theories. First-mode nonlinear frequency and time response of the viscoelastic microcantilever beam are analytically evaluated using multiple time scale method and then, validated through numerical findings. The obtained results indicate that nonlinear terms have an appreciable effect on natural frequency and time response of a viscoelastic microcantilever. Moreover, further investigations suggest that due to the size effects, natural frequency would drastically increase, especially when the thickness of the beam and the length scale parameter are comparable. The findings elaborate the significance of size effects in analyzing the mechanical behavior of small-scale structures.
\end{abstract}

(C) 2021 Sharif University of Technology. All rights reserved.

\section{Introduction}

Microstructures have considerably drawn researchers' attention in recent years due to their wide variety of applications. Among different types of microstructures, microbeams are highlighted due to their numerous industrial applications such as Microelectromechanical Systems (MEMS), capacitors, resonators, micro switches, atomic force microscopes, and biosensors.

\footnotetext{
*. Corresponding author. Tel.: +982166165503; Fax: +982166000021

E-mail address: Ahmadian@sharif.edu (M.T. Ahmadian)
}

doi: $10.24200 /$ sci. 2020.54137 .3612
Nevertheless, it is not surprising that the investigator focuses on the dynamical analysis of microbeams [1]. For instance, Ghommem and Abdelkefi [2] developed a nonlinear reduced-order model of an electrically actuated microcantilever beam with a tip mass using perturbation methods. They employed the resultant model as a resonant sensor for bio-mass detection and sensing, analyzed the nonlinear dynamics and effectiveness of the bio-mass sensor, and quantified the mass of biological entities.

On the contrary, numerous experimental results have proved the incapability of the classical theory to accurately predict the mechanical behavior of microscale beams. Studies on microscale structures showed that the classical theory could only estimate 
lower stiffness values, unlike experimental findings. The observations also indicated that the normalized deformation of microstructures depended on the size of the structure [3]. Due to the failure of classical theory in justifying and explaining the mechanical response of microscale structure, researchers proposed several nonclassical methods including Modified Strain Gradient (MSG) and Modified Coupled Stress (MCS) theories [4,5]. Mindlin [4] considered strain besides the first and second strain derivatives to formulate strain gradient theory. Fleck and Hutchinson [6] ignored the effect of the second derivative of the strain and reformulated and simplified the strain gradient theory. Lam et al. [7] modified the formulation derived from Fleck and Hutchinson's study [6] by employing a non-classical equilibrium equation (i.e., the equilibrium equation of the moment of couples) as well as the classical equilibrium equations (i.e., equilibrium equations of forces and moment of forces) [8].

A number of researchers utilized the strain gradient or the MCS theories to study the mechanical behavior of the microscale structures [9-13]. Chen and Li [14] studied buckling and post-buckling of the imperfect microbeam, analytically considering the mid-plane stretching for different boundary conditions. They examined the size effects through the modified coupled stress theory on the critical buckling, static response, and dynamic properties of imperfect microbeams. Akgöz and Civalek [15] derived formulations to bend the linear elastic microbeam based on MSG theory for different boundary conditions and analytically solved them. In addition, they studied buckling of an axially loaded microbeam and compared the calculated critical buckling load, which was based on the modified strain gradient theory, via the MCS theory [16]. In another research, Miandoab et al. [17] calculated the length scale parameters along with Young's modulus of polysilicone based on the MSG theory by fitting the experimental reported results of static pull-in voltages to the predicted ones.

Recently, the size effects on nonlinear vibration of viscoelastic microstructures have gained significance [18-22] since viscoelastic materials such as Polydimethylsiloxane (PDMS) [23] have been developed and used in manufactured microbeam biosensors due to their compliance and biocompatibility [24]. Li et al. $[25,26]$ formulated the governing equation of wave motion of fluid-conveying viscoelastic single-walled carbon nanotubes based on the nonlocal strain gradient theory. They discussed the importance of nonlocal as well as small-scale material parameters in the dispersion relation between the phase velocity and the wave number under magnetic field. Attia and Mohamed [27] considered the size effects based on the MCS theory and derived a non-classical nonlinear continuum model of an electrically actuated viscoelastic microbeams for different boundary conditions. Their model accounts for the nonlinearities due to axial residual stress, geometric nonlinearities, and electrical forcing with fringing effect. They modeled the viscoelasticity of the microbeam using Maxwell scheme and solved the resulting equations by the differential/integral quadrature model. In another research, they examined the effects of viscoelastic relaxation time, material length scale parameter, and size on the bending response of the Euler-Bernoulli nanobeams using nonlocal couple stress model [28]. They also studied free vibration of Functionally Graded (FG) viscoelastic nanobeams and investigated the effects of different parameters, including Poisson effect, surface elasticity, material length-scale, and material damping gradient index. They assessed the size effects based on MCS theory and formulated the viscoelasticity effect via the Kelvin Voigt model [29].

The oscillation of viscoelastic micro- and nanoscale Timoshenko beams based on fractional KelvinVoigt model was studied by Ansari et al. [30]. They employed Galerkin and predictor-corrector methods to analyze the impacts of length scale parameters, viscoelasticity coefficient, and fractional-order. The nonlinear dynamic stability of a viscoelastic microbeam was analyzed by Fu et al. [31]. They used numerical methods to solve the formulations and investigate the effects of creep quantity, geometric nonlinearity, environmental, and inner damping with symmetric electrostatic load on the principal region of instability. However, they did not take the length scale parameters into account. Fu and Zhang [32] examined the electromechanical dynamic buckling behavior of a viscoelastic microbeam. They also discussed the effects of length scale parameters via MCS theory to analyze the pull-in phenomenon of viscoelastic microbeams with electronic actuation [33]. Ghayesh and Farokhi studied the dynamics of Euler-Bernoulli viscoelastic microcantilevers such as stability and bifurcations [34,35], internal resonances and modal interactions [36], and large-amplitude oscillation $[37,38]$ and investigated the resonant response of an imperfect Timoshenko microbeam considering extensibility [39]. They employed the MCS theory to evaluate the effects of length scale size in these studies and numerically solved equations. In another study, Ghayesh [40] numerically analyzed the nonlinear mechanics of an elastically supported viscoelastic FG microcantilever considering energy transfer via internal resonance and motion complexity. He also employed the MCS theory to investigate the size effects.

A review of the studies on the vibration of nonlinear microcantilevers clearly shows that strain gradient size effects are not considered in modeling the nonlinear viscoelastic microcantilevers and the resultant formulations of full nonlinear models based on 
the MCS theory are numerically solved. In the present study, the general nonlinear equation of motion for a viscoelastic microcantilever beam was expressed based on the modified strain gradient theory represented by Lam et al. [7] and solved analytically for the first time. The nonlinear curvature effect and inertia terms were also taken into account while modeling the shortening effect. The equation was analytically solved through multiple time scale method, and timevariant nonlinear first-mode frequency of the microcantilever was consequently derived. According to the obtained results, the significance of size effects on the vibration amplitude, damping time, and natural frequency of microcantilever became more highlighted.

\section{Model development and analytical solution}

A typical rectangular beam of width $b$, thickness $h$, and length $L$ was considered in this study. For a nonlinear cantilever, the elongation of an element with length $d s$ (as depicted in Figure 1) can be defined as follows [41]:

$$
e=\frac{d s^{*}-d s}{d s}=\sqrt{\left(1+u^{\prime}\right)^{2}+v^{\prime 2}}-1
$$

where $u$ and $v$ are the longitudinal and lateral deflections of the cantilever, respectively, prime denotes derivative to $x$, and $z$ represents the distance of the cross-section from the neutral axis.

An inextensible beam was taken into account to model the shortening effect [41]:

$$
\begin{aligned}
e=0 & \Rightarrow u^{\prime}=\sqrt{1-v^{\prime 2}}-1 \approx-\frac{1}{2} v^{\prime 2} \\
& \Rightarrow u=-\int_{0}^{x} \frac{1}{2} v^{\prime 2} d x,
\end{aligned}
$$

Referring to Figure 1 [41]:

$$
\tan \theta=\frac{v^{\prime}}{1+u^{\prime}}
$$

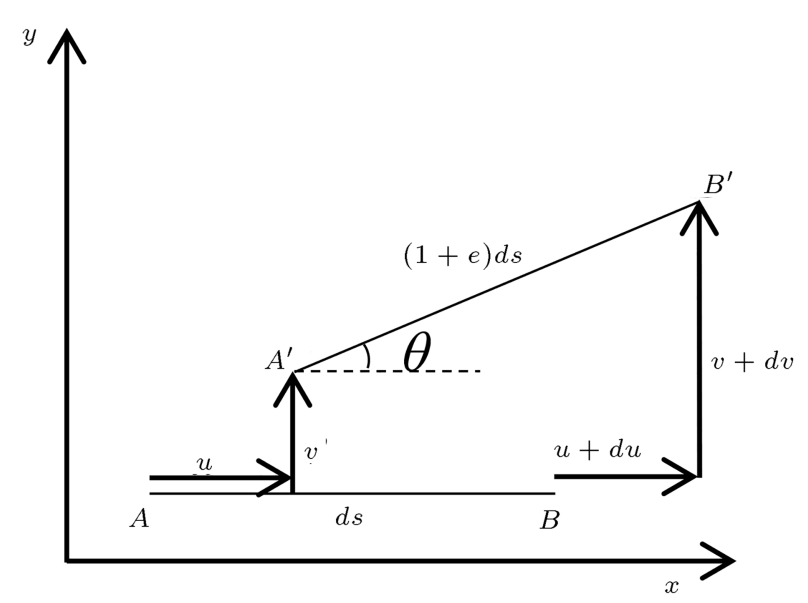

Figure 1. Coordinate system of a deflected microcantilever.
Differentiating Eq. (3) and using Eq. (2) would yield [41]:

$$
\theta^{\prime}=\frac{v^{\prime \prime}\left(1+u^{\prime}\right)-v^{\prime} u^{\prime \prime}}{\left(1+u^{\prime}\right)^{2}+v^{\prime 2}} \Rightarrow \theta^{\prime} \approx v^{\prime \prime}+\frac{1}{2} v^{\prime \prime} v^{\prime 2} .
$$

Hamilton's principle was employed to derive the equation of motion, according to which the kinetic and potential energy of vibrating homogenous microcantilever with a cross-section $A$ (i.e., $A=b h$ ) and density $\rho$ could be calculated.

The kinetic energy of the microcantilever can be obtained as shown in the following [41]:

$$
T=\frac{1}{2} \int_{0}^{L} \rho A\left\{\dot{u}^{2}+\dot{v}^{2}\right\} d x .
$$

By substituting $u$ into $v^{\prime}$ from Eq. (2) in Eq. (5), the following equation can be obtained [41]:

$$
T=\frac{1}{2} \int_{0}^{L}\left\{\rho A\left[\frac{\partial}{\partial t} \int_{0}^{x} \frac{1}{2}{v^{\prime}}^{2} d x\right]^{2}+\rho A \dot{v}^{2}\right\} d x .
$$

The density of strain energy of a linear elastic solid based on the MSG theory derived by Lam et al. [7] can be expressed as follows:

$$
\varrho=\sigma_{i j} \varepsilon_{i j}+p_{i} \gamma_{i}+\tau_{i j k}^{(1)} \eta_{i j k}^{(1)}+m_{i j}^{s} \chi_{i j}^{s},
$$

where the components of the classical strain tensor $(\varepsilon)$, dilatation gradient vector $(\gamma)$, deviatoric stretch gradient tensor $\left(\eta^{(1)}\right)$, and symmetric part of the rotation gradient tensor $\left(\chi^{s}\right)$ are defined as [7]:

$$
\begin{aligned}
\varepsilon_{i j}= & \frac{1}{2}\left(u_{i, j}+u_{j, i}\right), \\
\gamma_{i}= & \frac{\partial}{\partial i}\left(\varepsilon_{11}+\varepsilon_{22}+\varepsilon_{33}\right), \\
\eta_{i j k}^{(1)}= & \frac{1}{3}\left(\varepsilon_{i j, k}+\varepsilon_{j k, i}+\varepsilon_{k i, j}\right) \\
& -\frac{1}{15}\left[\delta_{i j}\left(\varepsilon_{m m, k}+2 \varepsilon_{m k, m}\right)\right. \\
& +\delta_{j k}\left(\varepsilon_{m m, i}+2 \varepsilon_{m i, m}\right) \\
& \left.+\delta_{k i}\left(\varepsilon_{m m, j}+2 \varepsilon_{m j, m}\right)\right], \\
\chi_{i j}^{s}= & \frac{1}{2}\left(e_{i p q} \varepsilon_{q j, p}+e_{j p q} \varepsilon_{q i, p}\right),
\end{aligned}
$$

where $u_{i}$ presents the components of the displacement vector $u$; and $\delta_{i j}$ and $e_{i j k}$ denote Kronecker delta and alternating tensor, respectively. For the viscoelastic material, the classical Kelvin-Voigt model is used. In this regard, the stress tensor $(\sigma)$ and the corresponding higher-order stresses $\left(p, \tau^{(1)}, m^{s}\right)$ relations are: 


$$
\begin{aligned}
& \sigma_{\text {tot }}=E \varepsilon+C \dot{\varepsilon}=\sigma+\sigma_{v i s}, \\
& p_{\text {tot }}=p+p_{v i s}=2 \mu_{k} l_{0}^{2} \gamma+2 \mu_{C} l_{0}^{2} \dot{\gamma} \\
& \tau_{\text {tot }}^{(1)}=\tau^{(1)}+\tau_{v i s}^{(1)}=2 \mu_{k} l_{1}^{2} \eta^{(1)}+2 \mu_{C} l_{0}^{2} \dot{\eta}^{(1)}, \\
& m_{\text {tot }}^{s}=m^{s}+m_{v i s}^{s}=2 \mu_{k} l_{2}^{2} \chi^{s}+2 \mu_{C} l_{2}^{2} \dot{\chi}^{s}
\end{aligned}
$$

where $E$ is the elastic modulus, $C$ is the damping coefficient, and $l_{0}, l_{1}, l_{2}$ are new material constants known as length scale parameters. Moreover, $\mu_{k}$ and $\mu_{C}$ are elastic and viscoelastic shear moduli related to Poisson's ratio $(v)$, as shown in the following:

$$
\mu_{k}=\frac{E}{2(1+v)}, \quad \mu_{C}=\frac{C}{2(1+v)} .
$$

The variation of works in terms of nonconservative forces can be formulated as:

$$
\delta W_{v i s}=-\int\left(\sigma_{v i s} \delta \varepsilon+p_{v i s} \delta \gamma+\tau_{v i s} \delta \eta+m_{v i s} \delta \chi\right) d V .
$$

The dimensionless parameters presented below are utilized to derive the normalized form of the differential equations:

$$
\begin{aligned}
& \bar{x}=\frac{x}{L}, \quad \bar{v}=\frac{v}{L}, \quad \theta=\sqrt{\frac{E I}{\rho A L^{4}}} \\
& \tau=\theta t, \quad \eta=\frac{C \theta}{E}, \quad \xi=\frac{\eta \omega}{2} \\
& S=E I \tilde{S}, \quad K=E I L^{2} \tilde{K} \\
& S_{C}=C I \tilde{S}, \quad K_{C}=C I L^{2} \tilde{K} \\
& \tilde{S}=1+\frac{6}{1+v}\left(2\left(\frac{l_{0}}{h}\right)^{2}+\frac{120}{225}\left(\frac{l_{1}}{h}\right)^{2}+\left(\frac{l_{2}}{h}\right)^{2}\right) \\
& \tilde{K}=\frac{1}{2(1+v)}\left(2\left(\frac{l_{0}}{L}\right)^{2}+\frac{4}{5}\left(\frac{l_{1}}{L}\right)^{2}\right)
\end{aligned}
$$

where $l$ is the area moment of inertia. The Hamilton principle can be used to achieve the normalized form of the nonlinear differential equation of motion and the related boundary conditions. It can be observed that due to the coupling effects of large deformation, strain gradient theory, and viscoelasticity, different nonlinearities are incorporated.

$$
\begin{aligned}
\frac{\partial^{2} \bar{v}}{\partial \tau^{2}}+ & +\tilde{S} \frac{\partial^{4} \bar{v}}{\partial \bar{x}^{4}}+\eta \tilde{S} \frac{\partial^{5} \bar{v}}{\partial \bar{x}^{4} \partial \tau}-\eta \tilde{K} \frac{\partial^{7} \bar{v}}{\partial \bar{x}^{6} \partial \tau}-\tilde{K} \frac{\partial^{6} \bar{v}}{\partial \bar{x}^{6}} \\
& +\tilde{S} \frac{\partial}{\partial \bar{x}}\left(\frac{\partial \bar{v}}{\partial \bar{x}} \frac{\partial}{\partial \bar{x}}\left(\frac{\partial \bar{v}}{\partial \bar{x}} \frac{\partial^{2} \bar{v}}{\partial \bar{x}^{2}}\right)\right) \\
& +\frac{\partial}{\partial \bar{x}}\left[\frac{\partial \bar{v}}{\partial \bar{x}} \int_{1}^{\bar{x}} \int_{0}^{s}\left(\left(\frac{\partial^{2} \bar{v}}{\partial \bar{x} \partial \tau}\right)^{2}+\frac{\partial \bar{v}}{\partial \bar{x}} \frac{\partial^{3} \bar{v}}{\partial \bar{x} \partial \tau^{2}}\right) d s d s\right]
\end{aligned}
$$

$$
\begin{aligned}
& -\eta \tilde{K}\left[\frac{\partial^{4}}{\partial \bar{x}^{3} \partial \tau}\left(\frac{\partial \bar{v}}{\partial \bar{x}} \frac{\partial}{\partial \bar{x}}\left(\frac{\partial \bar{v}}{\partial \bar{x}} \frac{\partial^{2} \bar{v}}{\partial \bar{x}^{2}}\right)\right)\right. \\
& -\frac{\partial}{\partial \bar{x}}\left(\frac{\partial^{2} \bar{v}}{\partial \bar{x} \partial \tau} \frac{\partial \bar{v}}{\partial \bar{x}} \frac{\partial^{5} \bar{v}}{\partial \bar{x}^{5}}\right)-\frac{\partial^{2}}{\partial \bar{x} \partial \tau}\left(\frac{\partial \bar{v}}{\partial \bar{x}} \frac{\partial^{2} \bar{v}}{\partial \bar{x}^{2}} \frac{\partial^{4} \bar{v}}{\partial \bar{x}^{4}}\right) \\
& \left.-\frac{\partial^{3}}{\partial \bar{x}^{2} \partial \tau}\left(\frac{\partial \bar{v}}{\partial \bar{x}} \frac{\partial^{2} \bar{v}}{\partial \bar{x}^{2}} \frac{\partial^{3} \bar{v}}{\partial \bar{x}^{3}}\right)\right] \\
& +\eta \tilde{S}\left[\frac{\partial^{2}}{\partial \bar{x} \partial \tau}\left(\frac{\partial \bar{v}}{\partial \bar{x}} \frac{\partial}{\partial \bar{x}}\left(\frac{\partial \bar{v}}{\partial \bar{x}} \frac{\partial^{2} \bar{v}}{\partial \bar{x}^{2}}\right)\right)\right. \\
& \left.-\frac{\partial}{\partial \bar{x}}\left(\frac{\partial^{2} \bar{v}}{\partial \bar{x} \partial \tau} \frac{\partial \bar{v}}{\partial \bar{x}} \frac{\partial^{3} \bar{v}}{\partial \bar{x}^{3}}\right)\right] \\
& -\tilde{K}\left[\frac{\partial^{3}}{\partial \bar{x}^{3}}\left(\frac{\partial \bar{v}}{\partial \bar{x}} \frac{\partial}{\partial \bar{x}}\left(\frac{\partial \bar{v}}{\partial \bar{x}} \frac{\partial^{2} \bar{v}}{\partial \bar{x}^{2}}\right)\right)\right. \\
& \left.-\frac{\partial^{2}}{\partial \bar{x}^{2}}\left(\frac{\partial \bar{v}}{\partial \bar{x}} \frac{\partial^{2} \bar{v}}{\partial \bar{x}^{2}} \frac{\partial^{3} \bar{v}}{\partial \bar{x}^{3}}\right)-\frac{\partial}{\partial \bar{x}}\left(\frac{\partial \bar{v}}{\partial \bar{x}} \frac{\partial^{2} \bar{v}}{\partial \bar{x}^{2}} \frac{\partial^{4} \bar{v}}{\partial \bar{x}^{4}}\right)\right] \\
& =0 \\
& {\left[\begin{array}{l}
\left.\left(\bar{v}^{\prime \prime \prime}+\overline{v^{\prime \prime \prime}}{\overline{v^{\prime}}}^{2}+\bar{v}^{\prime} \bar{v}^{\prime \prime}{ }^{2}\right) \delta \bar{v}\right|_{0} ^{1}=0 \\
\left.\left(\overline{v^{\prime \prime}}+\bar{v}^{\prime \prime}{\overline{v^{\prime}}}^{2}\right) \delta \bar{v}^{\prime}\right|_{0} ^{1}=0
\end{array}\right]} \\
& \Rightarrow\left[\begin{array}{l}
\bar{x}=0 \rightarrow \bar{v}=\bar{v}^{\prime}=0 \\
\bar{x}=1 \rightarrow \bar{v}^{\prime \prime}=\bar{v}^{\prime \prime \prime}=0
\end{array}\right] .
\end{aligned}
$$

The Galerkin method was employed so that the following mode shapes could be considered for the cantilever beam:

$$
\begin{aligned}
& \bar{v}=\sum_{m=1}^{\infty} \varphi_{m}(\bar{x}) q_{m}(\tau) \\
& \varphi_{m}(\bar{x})=\cosh \left(z_{m} \bar{x}\right)-\cos \left(z_{m} \bar{x}\right) \\
& \quad+\frac{\cos \left(z_{m}\right)+\cosh \left(z_{m}\right)}{\sin \left(z_{m}\right)+\sinh \left(z_{m}\right)}\left(\sin \left(z_{m} \bar{x}\right)-\sinh \left(z_{m} \bar{x}\right)\right), \\
& \cos \left(z_{m}\right) \cosh \left(z_{m}\right)+1=0 .
\end{aligned}
$$

The shape functions are normalized with the orthogonality condition of Eq. (24):

$$
<\varphi_{m}(\bar{x}), \varphi_{n}(\bar{x})>=\int_{0}^{1} \varphi_{m}(\bar{x}) \varphi_{n}(\bar{x}) d \bar{x}=\delta_{m n} .
$$

By substituting Eq. (21) into Eq. (19) and taking the inner product of the resulting equation with $\varphi_{1}$ (simply mentioned as $\varphi$ ), the first-mode shape timedependent equation can be expressed as the following differential equation:

$$
\begin{aligned}
& \ddot{q}+2 \xi \omega \dot{q}+\omega^{2} q+g_{1} q^{3}+g_{2} q \dot{q}^{2}+g_{3} q^{2} \ddot{q}+\frac{2 \xi g_{4}}{\omega} \dot{q} q^{2} \\
& =0
\end{aligned}
$$

where the coefficients $\omega$ and $g_{i}$ can be obtained as: 


$$
\begin{aligned}
& \omega^{2}=\tilde{S} a_{1}-\tilde{K} a_{2}, \\
& g_{1}=\tilde{S} a_{4}-\tilde{K} a_{5}, \\
& g_{2}=g_{3}=a_{3}, \\
& g_{4}=\tilde{S} a_{6}-\tilde{K} a_{7},
\end{aligned}
$$

where $a_{i}$ is derived as Eq. (27):

$$
\begin{aligned}
a_{1}= & \int_{0}^{1} \varphi \varphi^{I V} d \bar{x}, \\
a_{2}= & \int_{0}^{1} \varphi \varphi^{V I} d \bar{x}, \\
a_{3}= & \int_{0}^{1} \varphi\left(\varphi^{\prime} \int_{1}^{s} \int_{0}^{s} \varphi^{\prime 2} d s d s\right)^{\prime} d \bar{x}, \\
a_{4}= & \int_{0}^{1} \varphi\left(\varphi^{\prime \prime 3}+4 \varphi^{\prime} \varphi^{\prime \prime} \varphi^{\prime \prime \prime}+\varphi^{\prime 2} \varphi^{I V}\right) d \bar{x}, \\
a_{5}= & \int_{0}^{1} \varphi\left[\varphi^{\prime 2} \varphi^{V}+4 \varphi^{\prime} \varphi^{\prime \prime} \varphi^{I V}+6 \varphi^{\prime \prime 2} \varphi^{\prime \prime \prime}+3 \varphi^{\prime} \varphi^{\prime \prime \prime} 2\right] \\
& d \bar{x}, \\
a_{6}= & 3 a_{4}-\int_{0}^{1} \varphi\left[2 \varphi^{\prime} \varphi^{\prime \prime} \varphi^{\prime \prime \prime}+\varphi^{\prime 2} \varphi^{I V}\right] d \bar{x}, \\
a_{7}= & 3 a_{5}-\int_{0}^{1} \varphi\left[2 \varphi^{\prime} \varphi^{\prime \prime} \varphi^{V}+\varphi^{\prime 2} \varphi^{V I}\right] d \bar{x} .
\end{aligned}
$$

The multiscale perturbation method was employed to solve Eq. (25). For weak nonlinearities in the microcantilever beam, Eq. (25) can be rewritten as:

$$
\ddot{q}+2 \varepsilon \xi \omega \dot{q}+\omega^{2} q+\varepsilon N[q(\tau)]=0,
$$

where $N$ is the summation of nonlinear terms:

$$
N=g_{1} q^{3}+g_{2} q \dot{q}^{2}+g_{3} q^{2} \ddot{q}+\frac{2 \xi g_{4}}{\omega} \dot{q} q^{2} .
$$

To calculate the periodic solution of $q$, the expansion in terms of $\varepsilon$ can be written as:

$$
q=q_{0}\left(T_{0}, T_{1}\right)+\varepsilon q_{1}\left(T_{0}, T_{1}\right),
$$

where $T_{0}=\tau$ and $T_{1}=\varepsilon \tau$ are fast and slow time scales, respectively, that characterize the motions happening at the natural frequencies and related shifts due to nonlinearities. Then, in terms of the power of $\varepsilon$, Eq. (28) can be rewritten as follows:

$$
\varepsilon^{0} \rightarrow \frac{\partial^{2} q_{0}}{\partial T_{0}^{2}}+\omega^{2} q_{0}=0,
$$

$$
\begin{aligned}
\varepsilon^{1} \rightarrow & \frac{\partial^{2} q_{1}}{\partial T_{0}^{2}}+\omega^{2} q_{1}=-2 \frac{\partial^{2} q_{0}}{\partial T_{0} \partial T_{1}}-2 \xi \omega \frac{\partial q_{0}}{\partial T_{0}}-g_{1} q_{0}{ }^{3} \\
& -g_{2} q_{0}\left(\frac{\partial q_{0}}{\partial T_{0}}\right)^{2}-g_{3} q_{0}^{2} \frac{\partial^{2} q_{0}}{\partial T_{0}^{2}}-\frac{2 \xi g_{4}}{\omega} q_{0}^{2} \frac{\partial q_{0}}{\partial T_{0}} .
\end{aligned}
$$

While solving Eq. (31), $q_{0}$ can be determined as follows:

$$
q_{0}=A\left(T_{1}\right) e^{i \omega T_{0}}+\bar{A}\left(T_{1}\right) e^{-i \omega T_{0}} .
$$

Therefore, we have:

$$
\begin{aligned}
& q_{0}^{3}=A^{3} e^{3 i \omega T_{0}}+3 A^{2} \bar{A} e^{i \omega T_{0}}+C C, \\
& q_{0}\left(\frac{\partial q_{0}}{\partial T_{0}}\right)^{2}=\left(-\omega^{2}\right) \times\left(A^{3} e^{3 i \omega T_{0}}-A^{2} \bar{A} e^{i \omega T_{0}}+C C\right), \\
& q_{0}^{2} \frac{\partial^{2} q_{0}}{\partial T_{0}^{2}}=\left(-\omega^{2}\right) \times\left(A^{3} e^{3 i \omega T_{0}}+3 A^{2} \bar{A} e^{i \omega T_{0}}+C C\right), \\
& q_{0}^{2} \frac{\partial q_{0}}{\partial T_{0}}=(i \omega) \times\left(A^{3} e^{3 i \omega T_{0}}+A^{2} \bar{A} e^{i \omega T_{0}}+C C\right),
\end{aligned}
$$

where $C C$ denotes Complex Conjugate. By substituting Eq. (34) into Eq. (32), we have:

$$
\begin{aligned}
\frac{\partial^{2} q_{1}}{\partial T_{0}^{2}}+ & \omega^{2} q_{1}=-\left[g_{1}-\omega^{2}\left(g_{2}+g_{3}\right)+2 i \xi g_{4}\right] A^{3} e^{3 i \omega T_{0}} \\
& -\left\{\begin{array}{l}
2 i \omega A^{\prime}+2 i \xi \omega^{2} A+ \\
{\left[3 g_{1}+\omega^{2}\left(g_{2}-3 g_{3}\right)+2 i \xi g_{4}\right] A^{2} \bar{A}}
\end{array}\right\} e^{i \omega T_{0}} \\
& +C C
\end{aligned}
$$

In case the secular terms of Eq. (35) are equal to zero, $q_{1}$ can be calculated as:

$$
q_{1}=\frac{g_{1}-\omega^{2}\left(g_{2}+g_{3}\right)+2 i \xi g_{4}}{8 \omega^{2}} A^{3} e^{3 i \omega T_{0}}+C C .
$$

If the polar form of $A$ is used to omit secular terms of Eq. (35), we have:

$$
\begin{aligned}
A= & \frac{1}{2} \alpha\left(T_{1}\right) e^{i \beta\left(T_{1}\right)} \rightarrow \\
& \left\{\begin{array}{l}
\alpha^{\prime}+\xi \omega \alpha+\frac{\xi g_{4}}{4 \omega} \alpha^{3}=0 \\
\frac{\partial \beta}{\partial T_{1}}=\frac{3 g_{1}+\omega^{2}\left(g_{2}-3 g_{3}\right)}{8 \omega} \alpha^{2}
\end{array}\right\} .
\end{aligned}
$$

Calculating $\alpha$ and $\beta$ from Eq. (37) would result in:

$$
\left\{\begin{array}{l}
\alpha^{2}=\frac{4 \omega^{2} \alpha_{0}^{2}}{\left(4 \omega^{2}+g_{4} \alpha_{0}^{2}\right) e^{2 \xi \omega \varepsilon \tau}-g_{4} \alpha_{0}^{2}} \\
\beta=\frac{3 g_{1}+\omega^{2}\left(g_{2}-3 g_{3}\right)}{4 \xi g_{4}}\left(\ln \left(\begin{array}{l}
\left(4 \omega^{2}+g_{4} \alpha_{0}^{2}\right) e^{2 \xi \omega \varepsilon \tau} \\
-g_{4} \alpha_{0}^{2}
\end{array}\right)\right. \\
\left.-2 \xi \omega \varepsilon \tau-\ln \left(4 \omega^{2}\right)\right) .
\end{array}\right.
$$


By substituting Eq. (38) into Eq. (33) and Eq. (36), with consideration of Eq. (30) and Eq. (21), the nonlinear frequency and response of a viscoelastic microcantilever based on the MSG theory may be expressed as shown in the following:

$$
\left\{\begin{array}{l}
\omega_{N L}=\omega+\frac{\beta}{\tau} \\
v=\varphi\left[\alpha \cos \left(\omega_{N L} \tau\right)-\frac{\varepsilon \xi g_{4} \alpha^{3}}{16 \omega^{2}} \sin \left(3 \omega_{N L} \tau\right)\right. \\
\left.\quad+\frac{\varepsilon \alpha^{3}}{32 \omega^{2}}\left[\left(g_{1}-\omega^{2}\left(g_{2}+g_{3}\right)\right) \cos \left(3 \omega_{N L} \tau\right)\right]\right]
\end{array}\right.
$$

In the absence of damping, $\xi=0$, the outcome of Eq. (37) and Eq. (38) would be:

$$
\left\{\begin{array}{l}
\alpha=\alpha_{0} \\
\beta=\frac{3 g_{1}+\omega^{2}\left(g_{2}-3 g_{3}\right)}{8 \omega} \alpha_{0}^{2} \varepsilon \tau
\end{array}\right.
$$

if $l_{0}=l_{1}=l_{2}=0$, the obtained equation would be the same as that reported by Nayfeh and Nayfeh [42] for elastic microcantilever via classical beam theory.

$$
\left\{\begin{array}{l}
\omega_{N L}=\omega+\frac{\beta}{\tau} \\
v=\varphi\left\{\alpha \cos \left(\omega_{N L} \tau\right)+\frac{\varepsilon \alpha^{3}}{32 \omega^{2}}\left(g_{1}-\omega^{2}\left(g_{2}+g_{3}\right)\right)\right. \\
\left.\quad \cos \left(3 \omega_{N L} \tau\right)\right\}
\end{array}\right.
$$

\section{Results and discussion}

In this section, the first-mode nonlinear frequency and time response of the viscoelastic microcantilever beam are investigated considering the dimensionless parameters, namely $\eta=0.01, \nu=0.17, \omega_{1}=3.5160$, and $h / l=5$. Constants $a_{i}$ were evaluated for the first-mode shape of the microcantilever beam, and for MSG formulation, equal length scale parameters are considered (i.e., $l_{0}=l_{1}=l_{2}=l$ ) $[7,43]$.

The numerical results obtained from Eq. (28) were compared with the analytical findings from Eq. (38) plotted in Figure 2. The outcomes indicate that the two-term expansion of Eq. (38) models the time response fairly with the maximum error of $5 \%$.

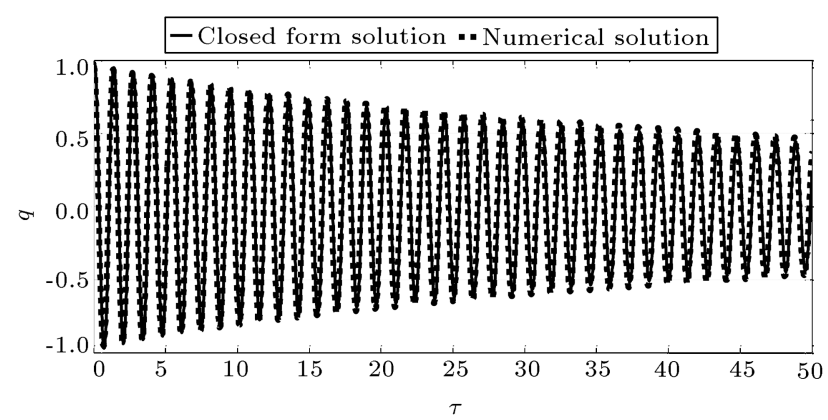

Figure 2. Numerical and two-term expansion model response of a viscoelastic microcantilever at the free end.

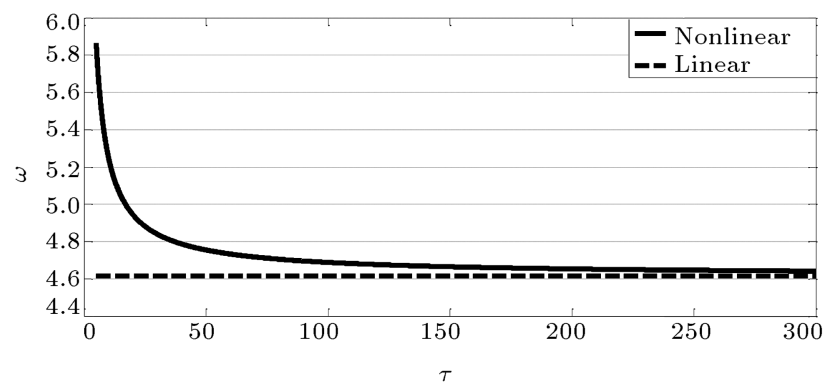

Figure 3. Linear and nonlinear frequencies of a viscoelastic microcantilever.

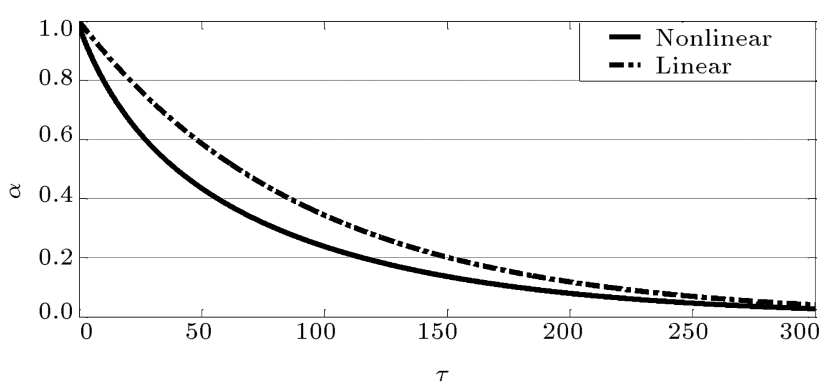

Figure 4. Linear and nonlinear maximum amplitudes of free vibration of a viscoelastic microcantilever.

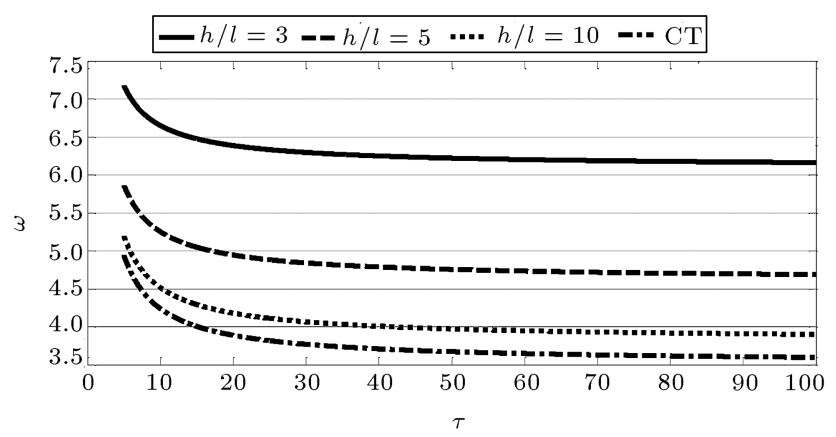

Figure 5. Nonlinear frequency of viscoelastic microcantilever for different values of $h / l$ and Classical Theory (CT).

The first mode linear and nonlinear natural frequencies of a viscoelastic microcantilever with respect to the MSG theory are depicted in Figure 3 which shows that nonlinear frequency approaches the linear one asymptotically over time. The dimensionless maximum amplitudes of linear and nonlinear vibrations are compared the results of which are shown in Figure 4. As observed, the nonlinear phenomena have a lower amplitude at a fixed frequency, implying that nonlinear effect causes higher strength in the structure.

The effects of length scale parameters on the firstmode frequency and the amplitude of viscoelastic microcantilever beam vibration are presented in Figures 5 and 6 . As the thickness of the microcantilever crosssection approaches the length scale parameters, the nonlinear frequency evaluated by MSG theory would increase (Figure 5). The same phenomenon for this 


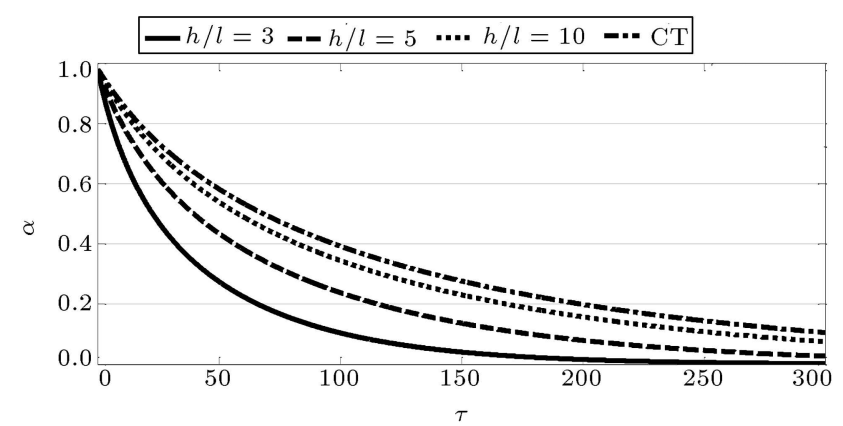

Figure 6. Nonlinear maximum amplitude for different values of $h / l$ and Classical Theory (CT).

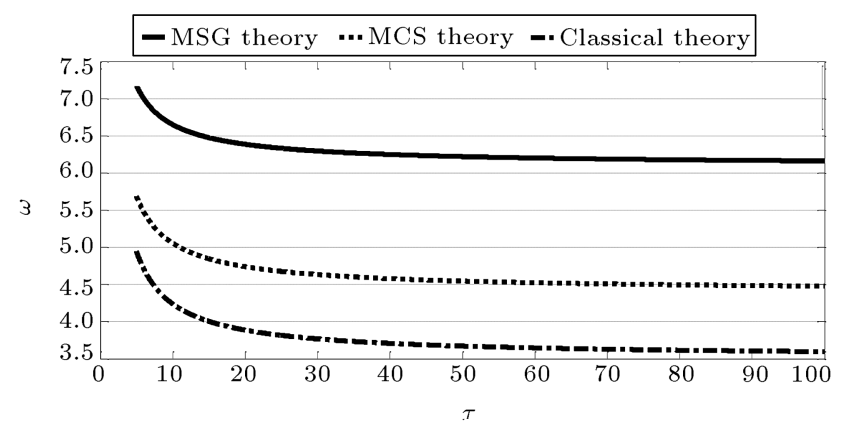

Figure 7. Nonlinear frequency of a viscoelastic microcantilever by Modified Coupled Stress (MCS) theory, Modified Strain Gradient (MSG) theory, and classical theory.

amplitude is depicted in Figure 6. The results indicated that as thickness approached the length scale, the vibration amplitude drastically decreased. Moreover, the hardening effect of considering the length scale parameters on the microcantilever behavior would lead to higher nonlinear frequency of the microcantilever and lower amplitude of the vibration. Figures 5 and 6 indicate that the effect of the length scale increased as the thickness-to-length scale parameter ratio of the beam reduced; therefore, the difference between the results of the derived model and predictions of the classical one cannot be ignored.

In Figures 7 and 8, the frequencies and maximum amplitudes of viscoelastic microcantilever beam versus time are compared based on the modified strain gradient, MCS, and classical theories. It can be observed that both MCS and MSG theories estimate higher stiffness for the beam than classical estimations; however, the modified strain gradient theory could improve the hardness much more than the MCS theory. In other words, the values of natural frequency calculated by the MSG theory are higher than those modeled by MCS theory, and the values estimated by MCS theory are higher than those estimated by the classical theory. However, the maximum amplitude of the free vibration was reversed, implying that the classical method could calculate the maximum amplitude of the vibration

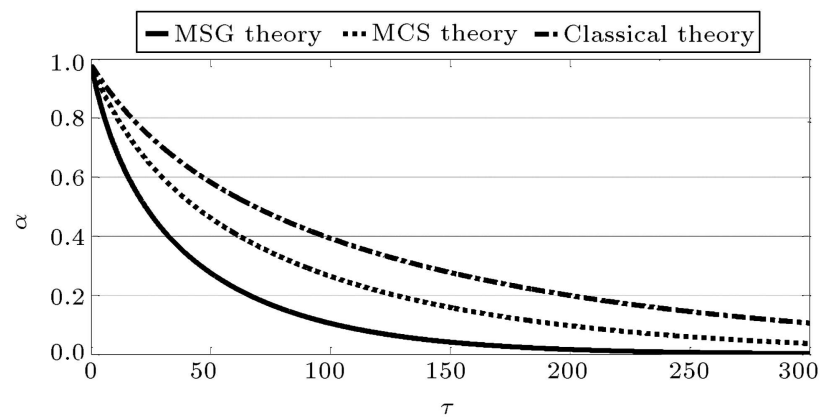

Figure 8. Maximum amplitude of nonlinear free vibration of a viscoelastic microcantilever by Modified Coupled Stress (MCS) theory, Modified Strain Gradient (MSG) theory, and classical theory.

more precisely than MCS theory, and MCS theory could calculate it more accurately than MSG theory.

\section{Conclusion}

A number of investigations have been conducted to model the nonlinear dynamics of microcantilevers. However, the size-dependent behavior of viscoelastic microcantilevers in the framework of strain gradient theory has not been analytically studied so far. In this paper, the nonlinear dynamics of the viscoelastic Euler-Bernoulli microcantilever beam via modified strain gradient theory was investigated using centerline inextensibility. To this end, the shortening effect theory was utilized to develop the equation of motion for the microcantilever beam. The Kelvin-Voigt scheme was also employed to model the viscoelastic behavior of the beam materials. The equation of motion was derived using Hamilton's principle, and the regular mode shape functions of a microcantilever were used to derive time response equation through the Galerkin method.

Through the perturbation method, i.e., the multiple time scales method, both nonlinear natural frequency and time response of the microcantilever were analytically obtained based on the modified strain gradient theory. The resultant formulations showed time dependency of the vibration amplitudes and nonlinear natural frequencies. This formulation allows modeling any nonlinearity arising from inertia, damping, and stiffness and directly derives analytical solutions for the first time.

The numerical results were measured to validate the findings. The first-mode nonlinear frequency and maximum amplitude of free vibration were compared using different methods such as the modified strain gradient, modified couple stress, and classical theories for various length scale parameters. The obtained results indicated that when the thickness of the beam was in order of the material length scale, the difference between the results of the classical and non-classical theories was considerable. However, as the thickness- 
to-material length scale ratio of the beam increased, the results of the non-classical assumption converged to those of the classical theory. These findings highlighted the significance of the size effect in analyzing the mechanical behavior of the small-scale structures.

\section{Acknowledgement}

This paper is supported by INSF.

\section{Nomenclature}

A Microbeam cross-section area

$b \quad$ Microbeam width

C Damping coefficient

$d s \quad$ Microbeam element length

$d V \quad$ Microbeam element volume

E $\quad$ Elastic modulus

e Microbeam element elongation

$e_{i j k} \quad$ Components of alternating tensor

$h \quad$ Microbeam thickness

$l \quad$ Area moment of inertia

$L \quad$ Microbeam length

$l_{0}, l_{1}, l_{2} \quad$ Length scale parameters

$m_{i j}^{s} \quad$ Components of higher-order stress due to the rotation gradient tensor

$p_{i} \quad$ Components of higher-order stress due to the dilatation gradient vector

$q(\tau) \quad$ The time part of the microbeam response

$T \quad$ The kinetic energy of the microbeam

$T_{0}, T_{1} \quad$ Fast and Slow dimensionless time scales

$u \quad$ Longitudinal deflection of the microbeam

$v$

$x$

$y$

$z$

$\chi_{i j}^{s}$

$\delta_{i j}$

$\delta W_{v i s}$

$\varepsilon_{i j}$

$\varphi(\bar{x})$

$\gamma_{i}$

$\begin{array}{ll}\eta_{i j k}^{(1)} & \begin{array}{l}\text { Components of deviatoric stretch } \\ \text { gradient tensor }\end{array} \\ \mu_{c} & \text { Viscoelastic shear modulus } \\ \mu_{k} & \text { Elastic shear modulus } \\ \rho & \text { Microbeam material density } \\ \sigma_{i j} & \text { Components of the classical stress } \\ \tau_{i j k}^{(1)} & \text { tensor } \\ v & \text { Components of higher-order stress due } \\ \omega & \text { to deviatoric stretch gradient tensor } \\ \theta & \text { Poisson's ratio } \\ & \text { Dimensionless frequency } \\ \text { Dot } & \text { The density of strain energy of the } \\ \text { Prime } & \text { Differentiation with respect to time } \\ & \text { Differentiation with respect to } x\end{array}$

\section{References}

1. Zand, M.M. and Ahmadian, M.T. "Application of homotopy analysis method in studying dynamic pullin instability of microsystems", Mechanics Research Communications, 36(7), pp. 851-858 (2009).

2. Ghommem, M. and Abdelkefi, A. "Nonlinear reducedorder modeling and effectiveness of electricallyactuated microbeams for bio-mass sensing applications", International Journal of Mechanics and Materials in Design, 15(1), pp. 125-143 (2019).

3. McFarland, A.W. and Colton, J.S. "Role of material microstructure in plate stiffness with relevance to microcantilever sensors", Journal of Micromechanics and Microengineering, 15(5), pp. 1060-1067 (2005).

4. Mindlin, R.D. "Second gradient of strain and surfacetension in linear elasticity", International Journal of Solids and Structures, 1(4), pp. 417-438 (1965).

5. Koiter, W.T. "Couple stresses in the theory of elasticity, I and II", Proceedings of the Koninklijke Nederlandse Akademie van Wetenschappen Series B, pp. 6717-6744 (1964).

6. Fleck, N.A. and Hutchinson, J.W. "A reformulation of strain gradient plasticity", Journal of the Mechanics and Physics of Solids, 49(10), pp. 2245-2271 (2001).

7. Lam, D.C.C., Yang, F., Chong, A.C.M., Wang, J., and Tong, P. "Experiments and theory in strain gradient elasticity", Journal of the Mechanics and Physics of Solids, 51(8), pp. 1477-1508 (2003).

8. Rahaeifard, M., Kahrobaiyan, M.H., Ahmadian, M.T., and Firoozbakhsh, K. "Strain gradient formulation of functionally graded nonlinear beams", International Journal of Engineering Science, 65, pp. 49-63 (2013).

9. Rahaeifard, M., Ahmadian, M., and Firoozbakhsh, K. "Vibration analysis of electrostatically actuated nonlinear microbridges based on the modified couple stress theory", Applied Mathematical Modelling, 39(21), pp. 6694-6704 (2015). 
10. Abbasi, M. and Mohammadi, A.K. "Study of the sensitivity and resonant frequency of the flexural modes of an atomic force microscopy microcantilever modeled by strain gradient elasticity theory", Journal of Mechanical Engineering Science, Proceedings of the Institution of Mechanical Engineers, Part C, 228(8), pp. 1299-1310 (2015).

11. Lazopoulos, A.K., Lazopoulos, K.A., and Palassopoulos, G. "Nonlinear bending and buckling for strain gradient elastic beams", Applied Mathematical Modelling, 38(1), pp. 253-262 (2014).

12. Karami, B., Shahsavari, D., Janghorban, M., and Li, L. "Influence of homogenization schemes on vibration of functionally graded curved microbeams", Composite Structures, 216, pp. 67-79 (2019).

13. Zhang, B., He, Y., Liu, D., Gan, Z., and Shen, L. "Non-classical Timoshenko beam element based on the strain gradient elasticity theory", Finite Elements in Analysis and Design, 79, pp. 22-39 (2014).

14. Chen, X. and Li, Y. "Size-dependent post-buckling behaviors of geometrically imperfect microbeams", Mechanics Research Communications, 88, pp. 25-33 (2018).

15. Akgöz, B. and Civalek, Ó. "Analysis of micro-sized beams for various boundary conditions based on the strain gradient elasticity theory", Archive of Applied Mechanics, 82(3), pp. 423-443 (2012).

16. Akgöz, B. and Civalek, Ö. "Strain gradient elasticity and modified couple stress models for buckling analysis of axially loaded micro-scaled beams", International Journal of Engineering Science, 49(11), pp. 1268-1280 (2012).

17. Miandoab, E.M., Yousefi-Koma, A., and Pishkenari, H.N. "Poly silicon nanobeam model based on strain gradient theory", Mechanics Research Communications, 62, pp. 83-88 (2014).

18. Mahmoodi, S., Khadem, S.E., and Kokabi, M. "Nonlinear free vibrations of Kelvin-Voigt visco-elastic beams", International Journal of Mechanical Sciences, 49(6), pp. 722-732 (2007).

19. Yazdi, F.C. and Jalali, A. "Vibration behavior of a viscoelastic composite microbeam under simultaneous electrostatic and piezoelectric actuation", Mechanics of Time-Dependent Materials, 19(3), pp. 277-304 (2015).

20. Zhu, C., Fang, X., and Liu, J. "A new approach for smart control of size-dependent nonlinear free vibration of viscoelastic orthotropic piezoelectric doublycurved nanoshells", Applied Mathematical Modelling, 77, pp. 137-168 (2020).

21. Zhu, C., Fang, X., and Yang, S. "Nonlinear free vibration of functionally graded viscoelastic piezoelectric doubly curved nanoshells with surface effects", The European Physical Journal Plus, 134(10), p. 486 (2019).

22. Zhu, C.-S., Fang, X.-Q., Liu, J.-X., and Li, H.$Y$. "Surface energy effect on nonlinear free vibration behavior of orthotropic piezoelectric cylindrical nanoshells", European Journal of Mechanics-A/Solids, 66, pp. 423-432 (2017).

23. Eduok, U., Faye, O., and Szpunar, J. "Recent developments and applications of protective silicone coatings: A review of PDMS functional materials", Progress in Organic Coatings, 111, pp. 124-163 (2017).

24. Le Digabel, J., Ghibaudo, M., Trichet, L., Richert, A., and Ladoux, B. "Microfabricated substrates as a tool to study cell mechanotransduction", Medical \& Biological Engineering \& Computing, 48(10), pp. 965976 (2010).

25. Li, L. and $\mathrm{Hu}, \mathrm{Y}$. "Wave propagation in fluidconveying viscoelastic carbon nanotubes based on nonlocal strain gradient theory", Computational Materials Science, 112, pp. 282-288 (2016).

26. Li, L., Hu, Y., and Ling, L. "Wave propagation in viscoelastic single-walled carbon nanotubes with surface effect under magnetic field based on nonlocal strain gradient theory", Physica E: Low-dimensional Systems and Nanostructures, 75, pp. 118-124 (2016).

27. Attia, M. and Mohamed, S. "Nonlinear modeling and analysis of electrically actuated viscoelastic microbeams based on the modified couple stress theory", Applied Mathematical Modelling, 41, pp. 195-222 (2017).

28. Attia, M. and Mahmoud, F. "Analysis of viscoelastic Bernoulli-Euler nanobeams incorporating nonlocal and microstructure effects", International Journal of Mechanics and Materials in Design, 13(3), pp. 385-406 (2017).

29. Attia, M.A. and Rahman, A.A.A. "On vibrations of functionally graded viscoelastic nanobeams with surface effects", International Journal of Engineering Science, 127, pp. 1-32 (2018).

30. Ansari, R., Oskouie, M.F., and Rouhi, H. "Studying linear and nonlinear vibrations of fractional viscoelastic Timoshenko micro-/nano-beams using the strain gradient theory", Nonlinear Dynamics, 87(1), pp. 695711 (2017).

31. Fu, Y., Zhang, J., and Bi, R. "Analysis of the nonlinear dynamic stability for an electrically actuated viscoelastic microbeam", Microsystem Technologies, 15(5), p. 763 (2009).

32. Fu, Y. and Zhang, J. "Electromechanical dynamic buckling phenomenon in symmetric electric fields actuated microbeams considering material damping", Acta Mech, 215(1-4), pp. 29-42 (2010).

33. Zhang, J. and Fu, Y. "Pull-in analysis of electrically actuated viscoelastic microbeams based on a modified couple stress theory", Meccanica, 47(7), pp. 1649-1658 (2012).

34. Ghayesh, M.H. "Stability and bifurcation characteristics of viscoelastic microcantilevers", Microsystem Technologies, 24(12), pp. 4739-4746 (2018). 
35. Ghayesh, M.H. and Farokhi, H. "Viscoelastically coupled size-dependent behaviour of imperfect extensible microbeams", International Journal of Mechanics and Materials in Design, 13(4), pp. 569-581 (2017).

36. Ghayesh, M.H. and Farokhi, H. "Size-dependent internal resonances and modal interactions in nonlinear dynamics of microcantilevers", International Journal of Mechanics and Materials in Design, 14(1), pp. 127140 (2018)

37. Ghayesh, M.H. and Farokhi, H. "Size-dependent largeamplitude oscillations of microcantilevers", Microsystem Technologies, 23(8), pp. 3477-3488 (2017).

38. Farokhi, H., Ghayesh, M.H., and Hussain, S. "Largeamplitude dynamical behaviour of microcantilevers", International Journal of Engineering Science, 106, pp. 29-41 (2016).

39. Farokhi, H. and Ghayesh, M.H. "Nonlinear resonant response of imperfect extensible Timoshenko microbeams", International Journal of Mechanics and Materials in Design, 13(1), pp. 43-55 (2017).

40. Ghayesh, M.H. "Mechanics of viscoelastic functionally graded microcantilevers", European Journal of Mechanics-A/Solids, 73, pp. 492-499 (2019).

41. Nayfeh, A.H. and Mook, D.T., Nonlinear Oscillations, John Wiley \& Sons (2008).

42. Nayfeh, A. and Nayfeh, S. "On nonlinear modes of continuous systems", Journal of Vibration and Acoustics, 116(1), pp. 129-136 (1994).

43. Kong, S., Zhou, S., Nie, Z., and Wang, K. "Static and dynamic analysis of micro beams based on strain gradient elasticity theory", International Journal of Engineering Science, 47(4), pp. 487-498 (2009).

\section{Biographies}

Farshad Taheran was born in Tehran, Iran in 1988. He received the BE degree in Mechanical Engineering from the University of Tehran, Tehran, Iran in 2010 and the MS degree in Mechanical Engineering from the Sharif University, Tehran, Iran in 2012. In master thesis, he worked on the vibration of drill strings, finding stability criteria. Since 2012, he has been a $\mathrm{PhD}$ student working at Sharif University, Tehran, Iran. His current research interests include vibration of viscoelastic microbeams based on strain gradient theory and stability conditions.

Mohammad Taghi Ahmadian received his BS and MS degrees in Physics in 1972 from Shiraz University, Shiraz, Iran and completed the requirements for these programs in Mechanical Engineering in 1980 from the University of Kansas in Lawrence. In the course of doing his master's degree, he completed his $\mathrm{PhD}$ program in Physics and PhD in Mechanical Engineering in 1981 and 1986, respectively, from the University of Kansas. His research interests are micro and nano-mechanics as well as Bioengineering.

Keykhosrow Firoozbakhsh received his $\mathrm{PhD}$ degree in Biomechanical Engineering (mechanical major) from Wayne State University, Detroit, Michigan, USA (1969-1972) and his Post Doctorate (bone remodeling) from Biomechanics Department, Tulane University, New Orleans, USA (1979-1980). At present, he is a Professor in the School of Mechanical Engineering at Sharif University of Technology, Tehran, Iran. His research interests are biomechanics and microstructures. 